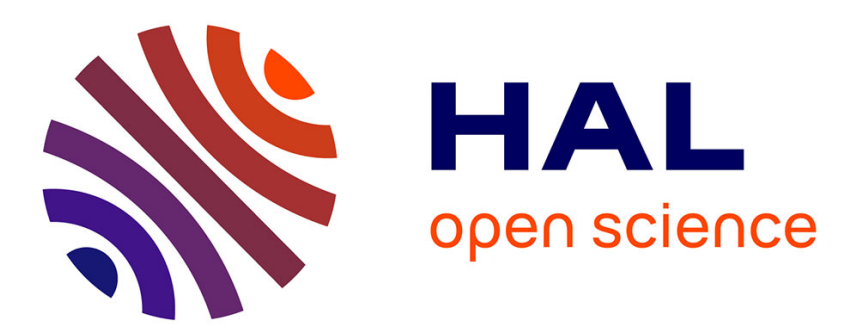

\title{
Electro-Optomechanical Modulation Instability in a Semiconductor Resonator
}

Pierre Etienne Allain, Biswarup Guha, Christophe Baker, David Parrain, Aristide Lemaître, Giuseppe Leo, Ivan Favero

\section{To cite this version:}

Pierre Etienne Allain, Biswarup Guha, Christophe Baker, David Parrain, Aristide Lemaître, et al.. Electro-Optomechanical Modulation Instability in a Semiconductor Resonator. Physical Review Letters, 2021, 126 (24), pp.243901. 10.1103/PhysRevLett.126.243901 . hal-03318926

\section{HAL Id: hal-03318926 \\ https://hal.science/hal-03318926}

Submitted on 11 Aug 2021

HAL is a multi-disciplinary open access archive for the deposit and dissemination of scientific research documents, whether they are published or not. The documents may come from teaching and research institutions in France or abroad, or from public or private research centers.
L'archive ouverte pluridisciplinaire HAL, est destinée au dépôt et à la diffusion de documents scientifiques de niveau recherche, publiés ou non, émanant des établissements d'enseignement et de recherche français ou étrangers, des laboratoires publics ou privés. 


\title{
Electro-optomechanical modulation instability in a semiconductor resonator
}

\author{
Pierre Etienne Allain, ${ }^{1}$ Biswarup Guha,${ }^{1}$ Christophe Baker, ${ }^{1}$ David \\ Parrain, ${ }^{1}$ Aristide Lemaître, ${ }^{2}$ Giuseppe Leo, ${ }^{1}$ and Ivan Favero ${ }^{1}$ \\ ${ }^{1}$ Matériaux et Phénomènes Quantiques, Université de Paris, \\ CNRS UMR 7162, 10 rue Alice Domon et Léonie Duquet 75013 Paris, France \\ ${ }^{2}$ Centre de Nanosciences et Nanotechnologies, CNRS UMR 9001, \\ Université Paris-Saclay, 91120 Palaiseau, France
}

(Dated: August 11, 2021)

\begin{abstract}
In semiconductor nano-optomechanical resonators, several forms of light-matter interaction can enrich the canonical radiation pressure coupling of light and mechanical motion, and give rise to new dynamical regimes. Here we observe an electro-optomechanical modulation instability in a Gallium Arsenide disk resonator. The regime is evidenced by the concomitant formation of regular and dense combs in the radio-frequency and optical spectrums of the resonator, associated with a permanent pulsatory dynamics of the mechanical motion and optical intensity. The mutual coupling between light, mechanical oscillations, carriers and heat, notably through photothermal interactions, stabilizes an extended mechanical comb in the ultra-high frequency range, which can be controlled optically.

PACS numbers: 78.40.Fy, 42.50.Wk, 42.65.Sf, 42.55.Sa, 85.85.+j
\end{abstract}

Storing light in a small cavity for a long time can enhance light-matter interactions. The approach has been employed to study the quantum electrodynamics of atoms [1], to engineer efficient lasers [2], or to investigate nonlinear optical phenomena, which emerge at large optical densities $[3,4]$. The richness of nonlinear optics is illustrated by various routes to chaos: under continuouswave laser driving and for an increasing power, the linear single-frequency output of a system can be substituted by a multi-frequency response, by the formation of temporal and spatial patterns, such as solitary waves, or by a chaotic type of response, where no apparent order surfaces [5]. A train of pulses usually precedes the emergence of optical solitons, a situation referred to as modulation instability $[6,7]$, which was observed in fibers, bulk crystals, semiconductor waveguide arrays, liquid crystals and dielectric cavities [8-10].

Mechanically compliant optical cavities may also confine light in order to enhance interactions, this time with a mechanical degree of freedom that replaces the atom, the gain or the nonlinear medium $[11,12]$. In high refractive index semiconductor optomechanical resonators, photons and phonons are co-localized in a sub-micron interaction volume, leading to large optomechanical couplings [13-15]. At this level of energy concentration, not only the optomechanical nonlinearity but other nonlinearities as well are expected to surge. In Gallium Arsenide (GaAs) disk resonators for example, a platform known for optomechanics [16, 17], the second-order optical nonlinearity was recently used for frequency conversion in high-Q whispering gallery resonators [18-21]. In Silicon photonic crystal resonators, the nonlinear optical absorption and thermo-optical response of the material were combined with optomechanical coupling in order to provide a route to chaos at large optical power [22, 23], which differed from that observed in the case of a mere radiation pressure optomechanical interaction [24]. Different regimes were observed in these experiments: optomechanical self-oscillations, self-induced optical modulation, generation of stable mechanical subharmonics in the optical output, and a fully developed chaos. Other permanent regimes may yet arise from the combination of various nonlinearities in a miniature optomechanical resonator, depending on the specific conditions met. This is the subject of the present paper.

Here we report on the observation of a regime of electro-optomechanical modulation instability in a GaAs disk resonator. The regime is evidenced by the concomitant formation of regular and dense combs in the radiofrequency and optical spectrums of the resonator, associated with a permanent pulsatory dynamics of the mechanical motion and optical intensity. A model, whose parameters have been carefully analyzed, quantitatively explains the emergence of this modulation instability and its evolution by the mutual couplings between the optical cavity field, the mechanical motion, and carriers and heat generated by linear and nonlinear optical absorption. The phenomenon settles at large optical power, beyond optomechanical self-oscillation, and enables the all-optical generation of a mechanical frequency comb in the ultra-high frequency range. The latter is stable and reaches a spectral span of one tenth of the carrier frequency.

Our GaAs disks are positioned in line on a mesa structure elevated above the substrate, as shown in Fig.1a. This allows using a straight tapered fiber for evanescent coupling experiments (Fig.1b), leading to a better control of polarization and mechanical stability compared to 
our past looped fiber technique $[14,16,25]$. The disks have a radius between 2 and $5 \mu \mathrm{m}$ and a thickness of 200 nm (Fig.1c). Resonators and mesa are fabricated out of an epitaxial GaAs/AlGaAs (Aluminum Gallium Arsenide) heterostructure using a combination of electronbeam and optical lithography and three distinct wet-etch steps. The disks are optically pumped through the fiber taper by a continuous-wave tunable single-mode laser operating at the telecom wavelength, while light at the fiber output is collected on a GHz-bandwidth photodetector. Under evanescent coupling, the disk mechanical motion modulates the fiber transmission, whose radio-frequency noise spectrum is analyzed electronically at the output of the photodetector.

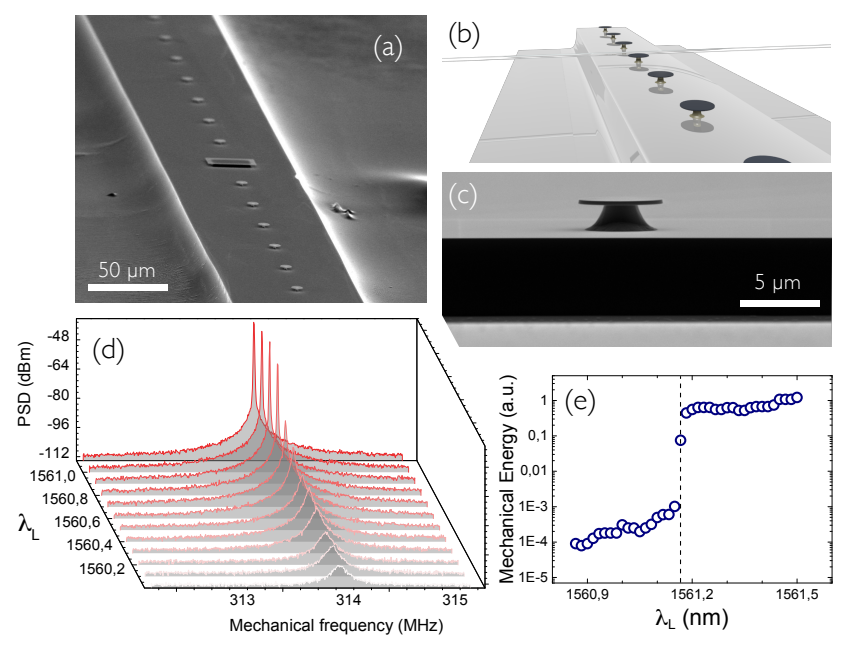

FIG. 1: GaAs disk optomechanical (self)-oscillators. (a) Electron micrograph of the mesa supporting a row of disk resonators. (b) Illustration of the straight fiber taper evanescently coupled to a disk of the row. (c) Side-view electron micrograph of a single disk. (d) Series of RF spectra showing the transition to optomechanical self-oscillation. (e) Threshold of the optomechanical self-oscillation regime as function of the driving wavelength.

Fig.1d shows the evolution of the obtained radiofrequency noise spectrum at moderate optical power in the fiber $(\sim 50 \mu \mathrm{W})$, for a laser blue-detuned on the flank of a whispering gallery mode (WGM1) resonance of a disk of radius $4.5 \mu \mathrm{m}$, as the laser wavelength is progressively increased to reduce the detuning (bottom to top curve). The bottom spectrum (white) displays a single peak at $314 \mathrm{MHz}$, corresponding to the fundamental radial breathing mode (RBM) of the disk driven by thermal fluctuations [16]. This peak is progressively narrowed and amplified before abruptly acquiring a large amplitude and a strongly reduced linewidth. In the time-domain, this corresponds to the onset of quasi-sinusoidal mechanical motion sustained by optomechanical forces, a situation often referred to as optomechanical (self-)oscillation
[26-29]. The oscillator transition and its threshold are better appreciated by plotting the area under the radiofrequency peak as a function of the driving laser wavelength (see Fig.1e), which controls both the detuning frequency and the number of intracavity photons, two key parameters of the amplification process $[11,12]$. In the specific case of the present GaAs disk operated at room temperature, the optomechanical self-oscillation is governed by a combination of radiation pressure, electrostriction and photothermal interactions, as detailed by experiments and a model elsewhere [30]. The latter model will be completed below in order to explain the richer dynamics developing at larger drive power, beyond optomechanical self-oscillation, which we now describe.
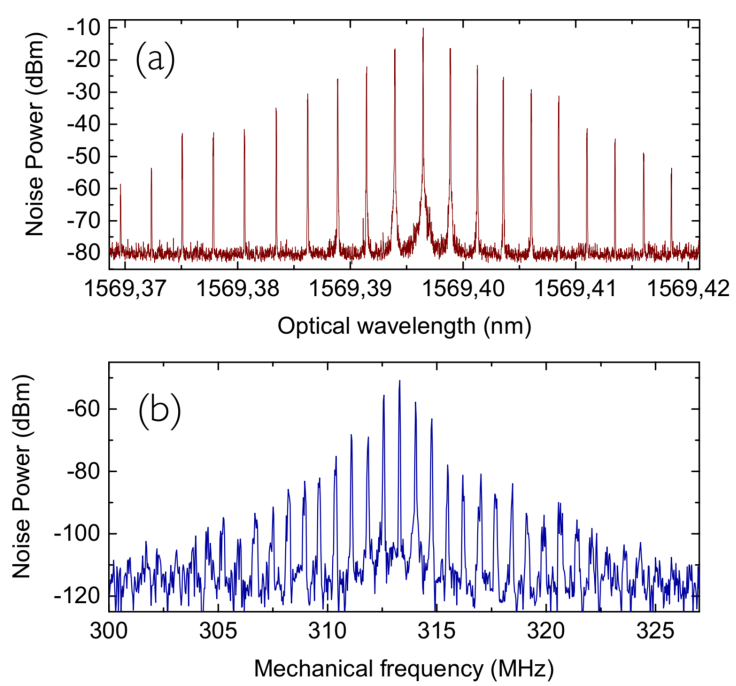

FIG. 2: Dual-comb structure in the electro-optomechanical modulation instability regime. (a) Optical spectrum of the light at the output of a GaAs disk resonator driven at large power. (b) Radio-frequency spectrum of the output light under the same conditions.

At larger power (typically $\geq 300 \mu \mathrm{W}$ ), the output light spectrum acquires a very regular comb pattern, both in the optical and radio-frequency domain. Fig.2a shows an example of the optical comb generated at the output of our GaAs disk resonator driven close to WGM1 resonance, measured with a high-resolution $(5 \mathrm{MHz})$ optical analyzer, with teeth separated by the mechanical frequency at $314 \mathrm{MHz}$. Several tens of teeth are visible in the spectrum, appearing as sidebands extending over a few tenths of picometers around the optical carrier wavelength at $1569.397 \mathrm{~nm}$. The formation of such optical comb is a feature known to accompany optomechanical self-oscillation, as reported in early work on the subject [27]. Less usual is in contrast the comb forming in the radio-frequency domain, visible in Fig.2b. Stable and regular, it is centered around the mechanical frequency and consists of about 30 visible teeth, with a frequency separation slightly below the $\mathrm{MHz}$ in this example. The 
latter comb formation, together with its exact spectral structure, is elucidated below by investigating the phenomenon in detail as function of the optical pump wavelength and power.

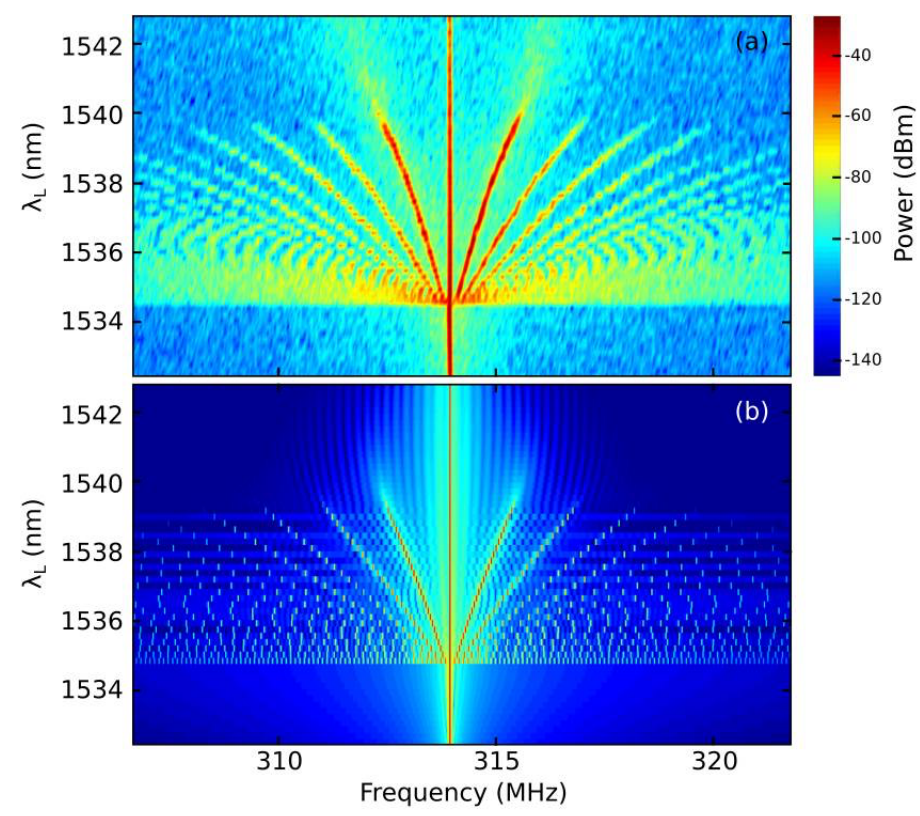

FIG. 3: Evolution of the electro-optomechanical modulation instability radio-frequency spectrum as function of the driving laser wavelength. (a) Experiment. (b) Theory.

Fig.3a follows the gradual evolution of a GaAs disk optomechanical resonator from the optomechanical selfoscillation regime to the double comb formation introduced above. The evolution is shown on a two-dimension map of the output radio-frequency spectrum as function of the optical drive wavelength $\lambda_{L}$. The resonator of 4.5 $\mu \mathrm{m}$ radius is driven on the blue-detuned flank of a second WGM optical resonance in the vicinity of $1530 \mathrm{~nm}$ of wavelength (WGM2), with an optical power of $3.56 \mathrm{~mW}$ in the fiber taper, and the laser detuning is progressively reduced as $\lambda_{L}$ is increased (bottom to top spectrum). $\lambda_{L}$ serves here as the control parameter of the evolution, but the optical power can be employed as well (not shown). At the starting wavelength in this map $\left(\lambda_{L} \sim 1533 \mathrm{~nm}\right)$, the spectrum consists of a single narrow line at $314 \mathrm{MHz}$, corresponding to the self-sustained optomechanical oscillation regime. At $\lambda_{L} \sim 1534.3 \mathrm{~nm}$, the spectrum suddenly develops a noisy background extending over 20 $\mathrm{MHz}$ around the mechanical frequency. As $\lambda_{L}$ is further increased, a very regular structure emerges from this background, with sidebands equally spaced and progressively incrementing in number. The frequency spacing between these teeth increases as $\lambda_{L}$ further increases, reaching above $1 \mathrm{MHz}$ at $\lambda_{L} \sim 1540 \mathrm{~nm}$. Beyond this point, these regular sidebands progressively fade away, letting again the self-oscillatory mechanical motion dom- inate the dynamics, with a single narrow peak in the spectrum.

In order to elucidate this behavior, we build on the optomechanical model introduced in [30], which includes radiation pressure, electrostrictive, and photothermal interactions. We extend the model by adding several nonlinearities: the two-photon absorption (TPA) of telecom photons leads to the generation of heat and free carriers. The heat increases the resonator temperature, which impacts intracavity photons through the thermo-optic effect. Photons are concomitantly impacted by free carriers through free-carrier dispersion (FCD) and absorption (FCA). The competition between these (slow) thermal and (fast) carrier nonlinearities is known to generate a rich radio-frequency dynamics in optical resonators [31]. Instead of the three coupled differential equations of [30] (optical, mechanical, thermal), we deal now with four coupled equations (optical, mechanical, thermal, free carriers). In contrast to $[22,23]$, our model includes the photothermal interaction (also dubbed bolometric force), which is a central ingredient in room temperature optomechanics [29, 30]: a mechanical displacement is generated by the thermal expansion following the absorption of photons, be it linear or nonlinear. The photothermal coupling transfers some of the thermal and optical dynamics to the mechanical degree of freedom, an important feature here. As shown below, this new model allows us to quantitatively reproduce the complex dynamics observed in our experiments. The four coupled equations read as follows:

$$
\begin{array}{r}
\dot{a}(t)=-\frac{\kappa}{2} a(t) \\
+i\left[\Delta^{b} \omega+g_{\text {om }} x(t)+\frac{\omega_{\text {cav }}}{n} \frac{d n}{d T} \Delta T(t)+\frac{\omega_{\text {cav }}}{n} \frac{d n}{d N} N(t)\right] a(t) \\
+\sqrt{\kappa_{e x}} a_{\text {in }}(t)
\end{array}
$$

$$
m_{\mathrm{eff}}\left[\ddot{x}(t)+\Gamma_{m} \dot{x}(t)+\omega_{m}^{2} x(t)\right]=F_{\text {opt }}(t)+F_{p t h}(t)
$$

$\dot{\Delta} T(t)=-\frac{\Delta T(t)}{\tau_{t h}}+\frac{R_{t h} \hbar \omega_{L}}{\tau_{t h}}\left(\kappa_{l i n}+\kappa_{\mathrm{TPA}}+\kappa_{\mathrm{FCA}}\right)|a(t)|^{2}$

$$
\dot{N}(t)=-\frac{N(t)}{\tau_{f c}}+\frac{\beta_{\mathrm{TPA}} c^{2} \hbar \omega_{L}}{2 n_{g}^{2} V_{\mathrm{FCA}}^{2}}|a(t)|^{4}
$$

Equation 1 governs the complex cavity optical field $a(t)$, normalized such that $|a(t)|^{2}$ is the number of cavity photons, $\kappa$ is the total cavity energy decay rate and $\kappa_{e x}$ the external coupling rate to the optical input field $a_{i n}$, normalized such that $\left|a_{i n}\right|^{2}=P_{L}$ is the input laser power. $\Delta^{b} \omega=\omega_{L}-\omega_{c a v}$ is the detuning of the laser frequency 
$\left(\omega_{L}=\frac{2 \pi c}{\lambda_{L}}\right)$ to the bare cavity resonance frequency $\left(\omega_{\text {cav }}\right)$, $g_{\text {om }}=-\frac{\partial \omega_{\text {cav }}}{\partial x}$ the frequency-pull parameter, $x(t)$ the mechanical displacement, $n$ a modal refractive index, $\frac{d n}{d T}$ the associated thermo-optic coefficient, $\Delta T$ the increase in disk temperature, $\frac{d n}{d N}$ the free-carrier dispersion coefficient with $N$ the density of free carriers. Equation 2 describes a mechanical harmonic oscillator of frequency $\omega_{m}$, effective mass $m_{\mathrm{eff}}$ and damping $\Gamma_{m}=\frac{\omega_{m}}{\mathrm{Q}_{m}}$, driven by conservative optical forces $F_{o p t}=F_{r p}+F_{\text {el }}$ (radiation pressure and electrostriction) summed with a dissipative photothermal force proportional to the temperature increase $F_{p t h}=\alpha \Delta T$. Equation 3 governs the thermal behavior of the resonator, with $\tau_{t h}$ the thermal relaxation time and $R_{t h}$ the thermal resistance that links, at steady-state, the temperature increase to the intracavity absorbed power $\Delta T=R_{t h}\left(\kappa_{l i n}+\kappa_{\mathrm{TPA}}+\kappa_{\mathrm{FCA}}\right) \hbar \omega_{L}|a|^{2}$. The intracavity absorption rate is the sum of a linear $\left(\kappa_{\text {lin }}\right)$, two-photon $\left(\kappa_{\mathrm{TPA}}\right)$ and free-carrier $\left(\kappa_{\mathrm{FCA}}\right)$ absorption rates, with $\kappa_{\mathrm{TPA}}=\left(\beta_{\mathrm{TPA}} c^{2} / V_{\mathrm{TPA}} n_{g}^{2}\right) \hbar \omega_{L}|a|^{2}$ and $\kappa_{\mathrm{FCA}}=\left(\sigma_{\mathrm{FCA}} c / n_{g}\right) N$, where $\beta_{\mathrm{TPA}}$ is the twophoton absorption coefficient (in $\mathrm{m} / \mathrm{W}$ ), $n_{g}$ is the groupvelocity index, $V_{\mathrm{TPA}}=\frac{\left(\int n^{2}(\mathbf{r})|E(\mathbf{r})|^{2} \mathrm{~d} \mathbf{r}\right)^{2}}{\int n^{4}(\mathbf{r})|E(\mathbf{r})|^{4} \mathrm{~d} \mathbf{r}}$ is the nonlinear volume and $\sigma_{\mathrm{FCA}}$ is the FCA cross section [32]. The total loss rate $\kappa=\kappa_{0}+\kappa_{e x}+\kappa_{\mathrm{TPA}}+\kappa_{\mathrm{FCA}}$ now includes several channels, with $\kappa_{0}=\kappa_{\text {lin }}+\kappa_{\text {rad }}$ the intrinsic decay rate of the cavity mode and $\kappa_{\text {rad }}$ the radiative contribution. Equation 4 describes the evolution of the density $N$ of free carriers generated by TPA. It involves the freecarrier relaxation time $\tau_{f c}$ and the FCA volume given by $V_{\mathrm{FCA}}^{2}=\frac{\left(\int n^{2}(\mathbf{r})|E(\mathbf{r})|^{2} \mathrm{~d} \mathbf{r}\right)^{3}}{\int n^{6}(\mathbf{r})|E(\mathbf{r})|^{6} \mathrm{~d} \mathbf{r}}[32]$. In the present work where the whispering gallery mode is mostly confined within the semiconductor material, the confinement factors $\Gamma_{\mathrm{TPA}}$ and $\Gamma_{\mathrm{FCA}}[32]$ have been omitted for simplicity.

The above set of four equations is closed and involves multiple parameters, which can be all evaluated independently by measurements or calculations. $\kappa_{e x}$ and $\kappa_{0}$ are measured through systematic evanescent coupling experiments with a varying gap [33]. $P_{L}, \Delta^{b} \omega, \Gamma_{m}, \omega_{m}$ and $g_{\text {om }}$ are measured through linear optical and mechanical spectroscopy [16], the latter parameter being also consistently obtained by Finite Element Method (FEM) simulations as the sum of a geometric $\left(g_{o m}^{g e o}\right)$ and a photoelastic $\left(g_{o m}^{p e}\right)$ contribution [15]. $n, d n / d T, d n / d N, \beta_{\mathrm{TPA}}$ and $\sigma_{\mathrm{FCA}}$ are calculated for the considered mode, starting from tabulated values under the here-employed telecom wavelength and room temperature conditions. The conservative forces are obtained through $F_{r p}=\hbar g_{o m}^{g e o}|a(t)|^{2}$ and $F_{e l}=\hbar g_{o m}^{p e}|a(t)|^{2}$, while the $\alpha$ coefficient of the photothermal force is computed using Eqs. 3.9 and 3.13 of [34]. Knowing the disk pedestal properties, $\tau_{t h}$ and $R_{t h}$ are computed by FEM, and we obtain consistently $\tau_{t h}$ from a frequency response measurement [35] and the product $\kappa_{\text {lin }} \times R_{t h}$ from a fit of the thermo-optic distortion of the WGM resonance at intermediate optical powers [30, 37]. Finally, $V_{\mathrm{TPA}}$ and $V_{\mathrm{FCA}}$ can be computed by FEM, while $\tau_{f c}$ is measured by time-resolved photoluminescence [38].

This lets the model with no adjustable parameter. In practice when running the model to fit the data, we did however let $\kappa_{l i n}, R_{t h}, \tau_{t h}, \alpha, \tau_{f c}, V_{\mathrm{TPA}}$ and $V_{\mathrm{FCA}}$ slightly vary around their first estimated value, within our estimation precision window. Table I lists the eventually employed parameter values, which all fall within this window. The only parameter showing some deviation is $\beta_{\text {TPA }}$, a factor nine above the bulk value generally reported in literature. This finding may be the result of the field confinement in our WGM resonators, which can modify the TPA by a few units [36], and of the presence of mid-gap states at the resonator surface [37, 38], which can assist nonlinear absorption [39]. Figure 3b shows the result of the modeling procedure, where the radiofrequency spectrum of the optical transmission is displayed as a function of laser wavelength. The model satisfactorily reproduces the experimental spectral response and all its salient features: the onset point of the comb formation is correctly retrieved, as well as its vanishing point, and the number of teeth together with their spectral separation are also precisely reproduced as a function of laser wavelength.

TABLE I: Parameters

\begin{tabular}{ccc}
\hline \hline Parameter & Value & Description \\
\hline$\omega_{\text {cav }}$ & $2 \pi \cdot 1.96 \times 10^{14} \mathrm{~Hz}$ & Bare cavity frequency \\
$\kappa_{0}$ & $2 \pi \cdot 1.25 \times 10^{10} \mathrm{~Hz}$ & Intrinsic cavity decay \\
$\kappa_{e x}$ & $2 \pi \cdot 1.61 \times 10^{9} \mathrm{~Hz}$ & Extrinsic cavity decay \\
$P_{L}$ & $3.56 \mathrm{~mW}$ & Input laser power \\
$g_{\text {om }}$ & $2 \pi \cdot 5.24 \times 10^{19} \mathrm{~Hz} \cdot \mathrm{m}^{-1}$ & Frequency-pull parameter \\
$d n / d T$ & $2.3 \times 10^{-4} \mathrm{~K}^{-1}$ & Thermo-optic coefficient \\
$d n / d N$ & $-5.53 \times 10^{-27} \mathrm{~m}^{3}$ & FCD coefficient \\
$\omega_{m}$ & $2 \pi \cdot 314 \mathrm{MHz}$ & Mechanical frequency \\
$m_{\mathrm{eff}}$ & $53 \mathrm{pg}$ & Effective mass \\
$\Gamma_{m}$ & $2 \pi \cdot 1.01 \times 10^{5} \mathrm{~Hz}$ & Mechanical damping \\
$\alpha$ & $1.9 \mu \mathrm{N} . \mathrm{K}^{-1}$ & Photothermal coefficient \\
$\tau_{t h}$ & $0.97 \mu \mathrm{s}$ & Thermal relaxation time \\
$R_{t h}$ & $3.78 \times 10^{6} \mathrm{~K} . \mathrm{W}^{-1}$ & Thermal resistance \\
$\kappa_{l i n}$ & $2 \pi \cdot 4.14 \times 10^{7} \mathrm{~Hz}$ & Linear absorption rate \\
$\beta_{\mathrm{TPA}}$ & $2.66 \times 10^{-9} \mathrm{~m} . \mathrm{W}^{-1}$ & TPA coefficient \\
$n_{g}$ & 2.6 & Group velocity index \\
$V_{\mathrm{TPA}}$ & $4.66 \times 10^{-18} \mathrm{~m}^{3}$ & Nonlinear TPA volume \\
$\sigma_{\mathrm{FCA}}$ & $6.03 \times 10^{-21} \mathrm{~m}^{2}$ & FCA cross-section \\
$\tau_{f c}$ & $14 \mathrm{ps}$ & Free-carrier relaxation time \\
$V_{\mathrm{FCA}}$ & $4.57 \times 10^{-18} \mathrm{~m}^{3}$ & FCA volume \\
\hline \hline
\end{tabular}

The electro-optomechanical modulation instability regime, where the radio-frequency comb is fully developed and stabilized, can also be observed by looking at the time response of the system. Fig.4a shows the optical transmission calculated from the above model under the conditions of Fig.3. On top of the expected modulation at the mechanical frequency of $314 \mathrm{MHz}$ (not visible at the scale of this plot), it displays a pronounced modu- 
lation of the envelope, both at fast $(\sim 10 \mathrm{~ns})$ and slow $(\sim 1.5$ and $0.5 \mu \mathrm{s})$ timescales. In order to appreciate the variety of the observed dynamics, we report in Fig.4b an experimental time trace of the fiber taper transmission obtained on the same resonator using a distinct optical mode (WGM3), whose spatial profile and coupling to free-carriers differ from those of WGM2. The time modulation is this time more symmetric and smoother, with a fast (slow) modulation time reaching time $\sim 100$ ns $(2.5 \mu \mathrm{s})$. Interestingly the temporal behavior of the mechanical displacement $x(t)$ in the modulation instability regime is also remarkably regular, as visible in the time trace of Fig.4c calculated from the model under the conditions of Fig.3. On top of the oscillation at $314 \mathrm{MHz}$ (not visible at this scale), it displays a very stable pulsing of the envelope at a slow timescale of $\sim 1 \mu \mathrm{s}$. The latter time can be varied with operating conditions, acting for example on the detuning or on the input power. In this regime, the spectrum of the mechanical motion acquires an extended frequency comb structure, which is shown in Figure 5.
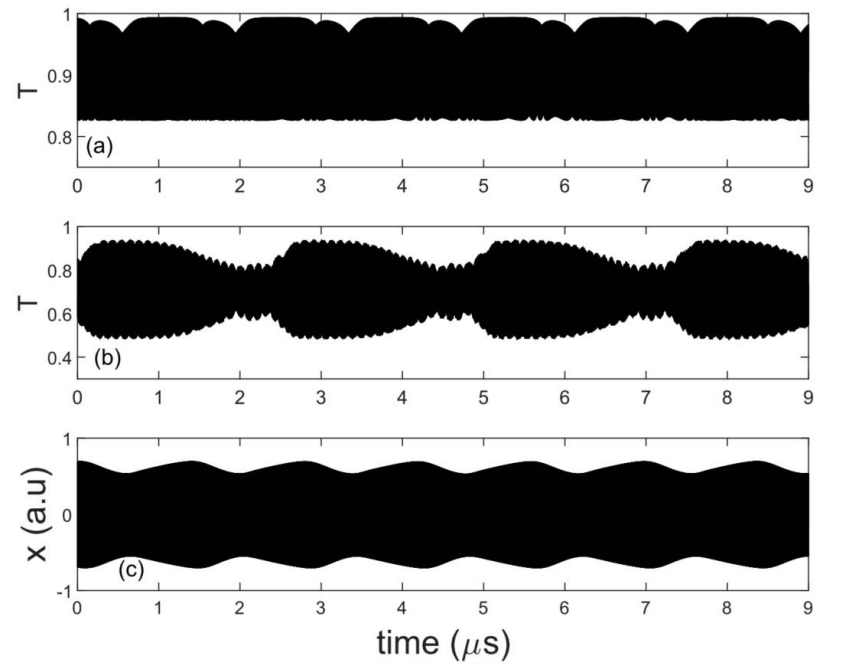

FIG. 4: Variety of the temporal dynamics met in the electrooptomechanical modulation instability regime. (a) Normalized transmission at the output of the fiber for WGM2, calculated from the model. (b) Time-trace of the normalized transmission at the output of the fiber for WGM3, measured experimentally. (c) Mechanical displacement as function of time under same conditions as (a).

In conclusion, we unravel here a new regular dynamical regime in optomechanical resonators, where carriers and heat interplay with canonical optomechanical coupling. Our experimental findings are precisely recovered using a model of four coupled equations, whose parameters have been carefully determined. The most remarkable experimental result is the stabilization of a dense and ordered $\mathrm{RF}$ comb around the mechanical carrier frequency, which manifests itself both in the spectrum of the output light and in the dynamics of the mechanical motion. The lat- ter acquires a very regular temporal behavior, where a smooth modulation of the envelope adds to the stable carrier frequency to generate a multi-frequency mechanical clock. The question arises if such mechanical frequency comb [40-42] can be made stable enough to be employed in force sensing $[35,43]$ with some advantage. Optomechanical magnetometers [44], atomic force [4547] or mass sensors [48, 49], may benefit from the development of mechanical comb spectroscopy controlled by light. Investigating these possibilities will require an indepth analysis of noise mechanisms in the here-presented electro-optomechanical oscillators, and will be the subject of future work.

This work was supported by the European Research Council via the GANOMS (306664) and NOMLI (770933) projects, and by the Agence Nationale de la Recherche through the Quantera QuaSeRT and Olympia projects.

[1] S. Haroche and J. M. Raimond, Exploring the quantum, Oxford University Press, England (2006).

[2] A. E. Siegman, Lasers, Oxford University Press, England (1986).

[3] N. W. Bloembergen, Nonlinear Optics, W. A. Benjamin, New York (1965).

[4] R. W. Boyd, Nonlinear Optics, Academic Press, Orlando, (2008).

[5] P. Bergé, Y. Pomeau, C. Vidal, L'ordre dans le Chaos, Hermann, Paris (1997).

[6] S. Wabnitz, Phys. Rev. A 38, 2018 (1988).

[7] B. Daino, G. Gregori, and S. Wabnitz, Journal of Applied Physics 58, 4512 (1985).

[8] G. Agrawal, Nonlinear Fiber Optics, Academic Press (1995).

[9] S. Trillo and W. Torruellas Eds., Spatial Solitons, Springer Series in Optical Sciences (2001).

[10] S. Barland, J. R. Tredicce, M. Brambilla, L. A. Lugiato, S. Balle, M. Giudici, T. Maggipinto, L. Spinelli, G. Tissoni, T. Knödl, M. Miller and R. Jäger, Nature 419, 699 (2002).

[11] I. Favero and K. Karrai, Nat. Phot. 3, 201-205 (2009).

[12] M. Aspelmeyer, T. J. Kippenberg and F. Marquardt, Review of Modern Physics 86, 1391 (2014).

[13] J. Chan, T. P. Mayer Alegre, A.H. Safavi-Naeini, J.T. Hill, A. Krause, S. Gröblacher, M. Aspelmeyer and O. Painter, Nature 478, 89 (2011).

[14] L. Ding, C. Baker, P. Senellart, A. Lemaître, S. Ducci, G. Leo and I. Favero, Appl. Phys. Lett. 98, 113108 (2011).

[15] C. Baker, W. Hease, D. T. Nguyen, A. Andronico, S. Ducci, G. Leo and I. Favero, Opt. Exp. 22, 14072 (2014).

[16] L. Ding, C. Baker, P. Senellart, A. Lemaître, S. Ducci, G. Leo and I. Favero, Phys. Rev. Lett. 105, 263903 (2010).

[17] L. Ding, C. Baker, A. Andronico, D. Parrain, P. Senellart, A. Lemaître, S. Ducci, G. Leo and I. Favero. GaAs disk optomechanical resonators, in Handbook of optical microcavities, PanStanford (2014).

[18] A. Andronico, I. Favero and G. Leo, Opt. Lett. 33, 2026 (2008). 
[19] P. S. Kuo, J. Bravo-Abad and G. S. Solomon, Nat. Comm. 5, 3109 (2014).

[20] S. Mariani, A. Andronico, A. Lemaître, I. Favero, S. Ducci, and G. Leo, Opt. Lett. 39, 3062 (2014).

[21] I. Roland, A. Borne, M. Ravaro, R. De Oliveira, S. Suffit, P. Filloux, A. Lemaître, I. Favero, and G. Leo, Optics Letters 45, 2878 (2020).

[22] D. Navarro-Urrios, N. E. Capuj, M. F. Colombano, P. D. Garcia, M. Sledzinska, F. Alzina, A. Griol, A. Martinez, and C. M. Sotomayor-Torres, Nat. Commun. 8, 14965 (2017).

[23] J. Wu, S. W. Huang, Y. Huang, H. Zhou, J. Yang, J. M. Liu, M. Yu, G. Lo, D. L. Kwong, S. Duan, and C. W. Wong, Nat. Commun. 8, 15570 (2017).

[24] T. Carmon, M. C. Cross, and K. J. Vahala, Phys. Rev. Lett. 98, 167201 (2007).

[25] L. Ding, C. Belacel, S. Ducci, G. Leo and I. Favero, Applied Optics 49, 2441 (2010).

[26] C. Höhberger Metzger and K. Karrai, Nature 432, 1002 (2004).

[27] T. Carmon, H. Rokhsari, L. Yang, T. J. Kippenberg and K. J. Vahala, Phys. Rev. Lett. 94, 223902 (2005).

[28] F. Marquardt, J. G. E. Harris and S. M. Girvin, S. M. Phys. Rev. Lett. 96, 103901 (2006).

[29] C. Metzger, M. Ludwig, C. Neuenhahn, A. Ortlieb, I. Favero, K. Karrai, and F. Marquardt. Phys. Rev. Lett. 101, 133903 (2008).

[30] B. Guha, S. Mariani, A. Lemaître, S. Combrié, G. Leo, and I. Favero. Optics Express 25, 24639 (2017).

[31] T. J. Johnson, M. Borselli, and O. Painter. Optics Express 14, 817 (2006).

[32] P. E. Barclay, K. Srinivasan, and O. Painter. Optics Express 13, 801 (2005).

[33] L. Ding, P. Senellart, A. Lemaitre, S. Ducci, G. Leo, and I. Favero. Proc. SPIE 7712 , 771211 (2010).

[34] I. Favero. Optomechanical Interactions, in Quantum Optomechanics and Nanomechanics Lecture Notes of the Les Houches Summer School, Oxford University Press (2020).

[35] B. Guha, P. E. Allain, A. Lemaître, G.Leo, and I. Favero. Physical Review Applied 14, 024079 (2020).

[36] A. Baron, A. Ryasnyanskiy, N. Dubreuil, P. Delaye, Q. Vy Tran, S. Combrié, A. de Rossi, R. Frey, and G. Roosen. Optics Express 17, 552 (2009).

[37] D. Parrain, C. Baker, G. Wang, A. Lemaitre, P. Senellart, G. Leo, S. Ducci and I. Favero. Optics Express 23, 19656 (2015).

[38] B. Guha, F. Marsault, F. Cadiz, L. Morgenroth, V. Ulin, V. Berkovitz, A. Lemaître, C. Gomez, A. Amo, S. Combrié, B. Gérard, G. Leo and I. Favero. Optica 4, 218 (2017).

[39] Z. Zhou, C. Lu, S. Xu, Y. Jiang, B. Yun, C. Wang, and Y. Cui. Applied Physics Letters 103, 231111 (2013).

[40] A. A. Savchenkov, A. B. Matsko, V. S. Ilchenko, D. Seidel, and L. Maleki. Opt. Lett. 36, 3338 (2011).

[41] A. Ganesan, C. Do, and A. Seshia. Phys. Rev. Lett 118, 033903 (2017).

[42] D. A. Czaplewski, C. Chen, D. Lopez, O. Shoshani, A. M. Eriksson, S. Strachan, and S. W. Shaw. Phys. Rev. Lett 121, 244302 (2018).

[43] L. Mercier de Lépinay, B. Pigeau,, B. Besga, P. Vincent, P. Poncharal, and O. Arcizet. Nat. Nanotech. 12, 156 (2017).

[44] S. Forstner, S. Prams, J. Knittel, E. D. van Ooijen, J. D.
Swaim, G. I. Harris, A. Szorkovszky, W. P. Bowen, and H. Rubinsztein-Dunlop. Phys. Rev. Lett. 108, 120801 (2012).

[45] J. Chae, S. An, G Ramer, V. Stavila, G. Holland, Y. Yoon, A. A. Talin, M. Allendorf, V. A Aksyuk, and A. Centrone. Nanoletters 17, 5587 (2017).

[46] P. E. Allain, L. Schwab, C. Mismer, M. Gely, E. Mairiaux, M. Hermouet, B. Walter, G. Leo, S. Hentz, M. Faucher, G. Jourdan, B. Legrand, and I. Favero. Nanoscale 12, 2039 (2020).

[47] D. Hälg, T. Gisler, Y. Tsaturyan, L. Catalini, U. Grob, M. D. Krass, M. Héritier, H. Mattiat, A. K. Thamm, R. Schirhagl, E. C. Langman, A. Schliesser, C. L. Degen, and A. Eichler. Physical Review Applied 15, 021001 (2021)

[48] W. Yu, W. C. Jiang, Q. Lin, T. Lu. Nat. Commun. 7, 12311 (2016).

[49] M. Sansa, M. Defoort, A. Brenac, M. Hermouet, L. Banniard, A. Fafin, M. Gely, C. Masselon, I. Favero, G. Jourdan, and S. Hentz. Nat. Commun. 11, 3781 (2020).

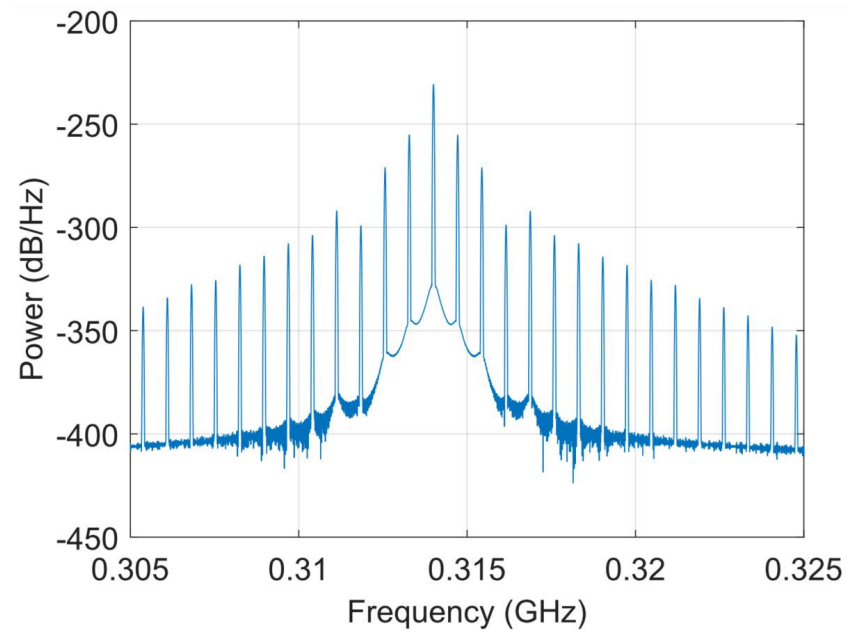

FIG. 5: Mechanical comb controlled by light. Spectrum of the pulsing mechanical motion shown in Fig. 4c of the main text, obtained by Fast Fourier Transform using a Kaiser window. 

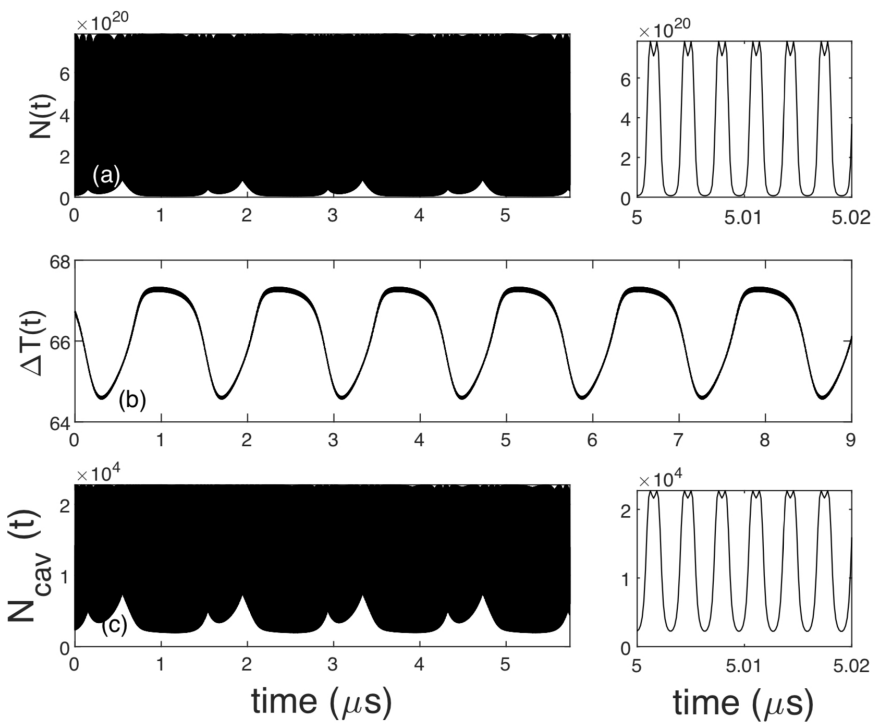

FIG. 6: Time evolution of the variables involved in the electrooptomechanical instability. (a) Free-carrier density, (b) Temperature elevation, (c) Intracavity photon number, as a function of time. Time traces are calculated from the model introduced in the main text, under the conditions of Fig. 3. 